\title{
Flaxseed oil (Linum usitatissimum) attenuates restraint stress-induced depressive-like behavior: Upregulation of neurotrophic factors in CA1 region of hippocampus
}

\author{
Sahand Talei ${ }^{1}$, Tahmineh Mokhtari², Ilia Asadi³, Leila Arab , Negar Hassanzadeh5 , Emadoddin Hosseinjani ${ }^{5}$, \\ Gholamreza Hassanzadeh ${ }^{6,7,8 *}$
}

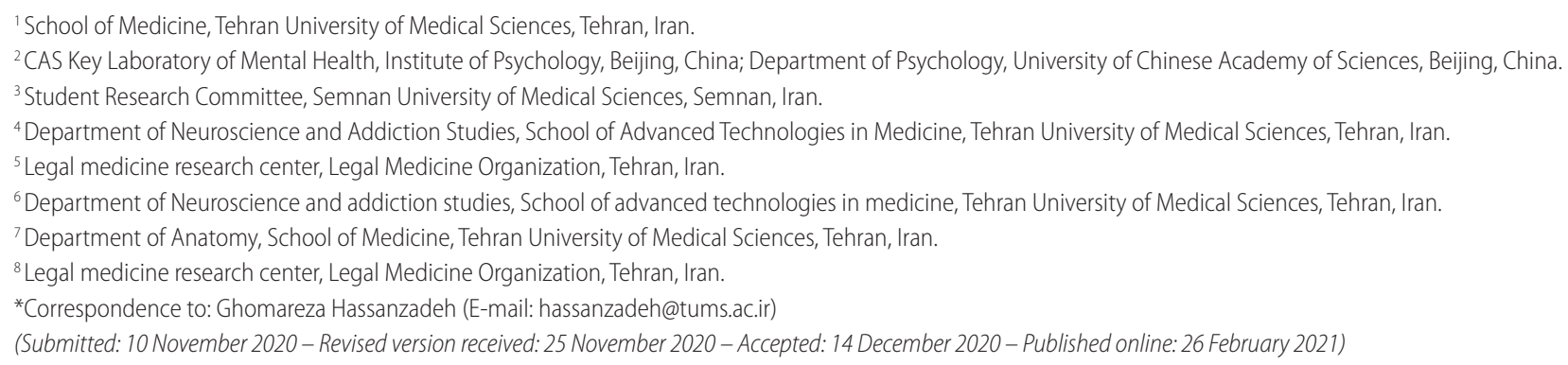

\section{Introduction}

Nowadays, the human life style cause a lot of stress for individuals which could be ended to depression and anxiety disorders. ${ }^{1}$ Stress affect different body organs and their functions and also increase the level of cortisol in blood. High level of cortisol decreases the muscle mass, increases the blood glucose and body fat, induces insomnia, anorexia, fatigue, and brain atrophy. ${ }^{2,3}$ The neurotrophins including brain-derived neurotrophic factor (BDNF) and glial cell line-derived neurotrophic factor (GDNF) have important roles in central nervous system like neuroprotective effects against neuroinflammation and oxidative toxicity that help the neurons to survive, maintain, migration, and differentiation., ${ }^{4}$ But, as a consequence of stress, these neurotrophic factors (NTFs) decrease and cause mood disorders specially depression., 7 NTFs, including BDNF and GDNF, are involved in the different steps of development and function of neurons. ${ }^{8}$ Previous studies demonstrated that the level of BDNF and GDNF was significantly lower than healthy individuals. ${ }^{9,} 10$ Exposure to the chronic stress induced hippocampal atrophy and decrease of neurogenesis in hippocampus and impairments in cognition and memory. ${ }^{11-13}$ Impairment of serotonergic system lead to depression and anxiety disorders. BDNF and GDNF stimulate the growth of 5-HT neurons and also have direct effect on gene expression in serotonergic system. ${ }^{14}$

Various drug treatments are used in anxiety disorders and depression but the harms of side effects for the patients lead to evaluation of herbal drugs as a natural harmless treatment. Flaxseed (FS, Linum usitatissimum) is a 1-year planet which is a valuable source of phenol complex, antioxidant agents, alpha-linolenic acid (ALA), fibers, minerals, and vitamins. ${ }^{15,16}$ The ALA of FS oil increase the expression of BDNF gene and related mRNA in hippocampus. In rats with middle cerebral artery occlusion, nutrition with FS, increase the BDNF and GDNF gene expression in motor cortex and CA1 area of hippocampus. ${ }^{15}$ According to decrease of BDNF and GDNF in depression, ${ }^{17}$ we designed this study to evaluate the effect of FS oil on BDNF and GDNF gene expression in CA1 area of hipocampus in mice with immobility stress.

\section{Method and Materials}

\section{Animals}

In this study, 32 white laboratory male mice (20-25 g, 12-week old) were enrolled. They were kept in a room with temperature of $18-24^{\circ} \mathrm{C}, 40-70 \%$ humidity, $12 \mathrm{~h}$ light-12h dark cycle, and 
free access to food and water. They were treated according to the guidelines of Iranian council for use and care of animals and approved by Ethical Committee of Tehran University of Medical Science.

The mice were randomly divided in 4 groups with 15 mice:

Group 1: Control; the mice were fed with water and routine food for 20 days without any stress or treatment.

Group 2: Flaxseed (FS) oil; mice were fed with water and routine food, and they also received FS oil $(0.2 \mathrm{ml} /$ daily by gavage) for 20 days without any stress.

Group 3: Immobility stress model (Model); mice with depressive-like behavior (immobility stress for $6 \mathrm{~h} /$ day for 20 days) and feeding with normal saline by oral gavage for another 20 days.

Group 4: Model+FS oil; mice with depressive-like behavior (immobility stress for $6 \mathrm{~h}$ /day for 20 days) and FS oil $(0.2 \mathrm{ml} /$ daily by gavage $)$ for another 20 days.

\section{Immobility stress induction}

For model induction, the mice were kept in $50 \mathrm{ml}$ Falcon tubes which made them totally immobile for $6 \mathrm{~h}$ a day. During this period, other stressors like noise, light, or temperature changes were omitted. At the end of induction period, the mice were returned to their cage. After 20 days, all the mice were sacrificed by cardiac puncture under anesthesia to extract their whole brain for more evaluation.

\section{Brain tissue sampling}

Fresh samples of hippocampus were extracted immediately after sacrificing and put in freezing tube and kept in $-80^{\circ} \mathrm{C}$. For histological studies, the brains were prefixed by a transcardial perfusion of normal saline (NS) followed by $4 \%$ paraformaldehyde (PFA, Sigma in $0.1 \mathrm{M}$ phosphate buffer pre-fixation). The post-fixed was done by using $10 \%$ formalin at $4^{\circ} \mathrm{C}$ for $72 \mathrm{~h}$.

\section{Hematoxylin \& eosin staining}

After fixation and tissue processing, the 5 um-thick coronal sections were prepared by rotary microtome (Leica Biosystems, Milan, Italy). For light microscopy observation, the tissue sections stained with Hematoxylin and eosin (H \& E) according to the standard protocol and analyzed via a light field microscope (Olympus, CX31, Tokyo, Japan). The photomicrographs were prepared from CAl region and the number of dead neurons (eosinophilic neurons) were calculated in each photomicrographs.

\section{Total RNA extraction and quantitative real-time PCR}

Gene expression of BDNF and GDNF were evaluated after $24 \mathrm{~h}$ of sacrificing. Under anesthesia, the brain was extracted and placed in ice-cold saline $(0.9 \%)$. Then, the hippocampal CA1 region was isolated. The expression of BDNF and GDNF genes in CA1 region was assessed by quantitative real-time PCR (ABI PRISM 7500 real-time PCR system, Roche Diagnostics, Germany). The total RNA was extracted, and cDNA was synthesized from $1 \mu \mathrm{g}$ total RNA using PrimeScript RT Reagent Kit (Takara Bio Inc., Otsu, Japan). Quantitative real-time PCR was performed in a Cycler (Light Cycler 2.0, Roche) by SYBR Green (Takara Bio Inc., Otsu, Japan). The primers were designed using Generunner software (Table 1). The b-actin gene was as the internal control standard.

\section{Protein concentration}

The protein concentrations of BDNF and GDNF in hippocampal CA1 region were assessed by ABCAM ELISA kits in accordance with their manufacturer's guidelines. Hippocampus CA1 area was homogenized and after the centrifuge $\left(14,000 \mathrm{rpm}, 4^{\circ} \mathrm{C}\right.$ for $3 \mathrm{~min}$ ), the supernatant was diluted with sample buffer. Then, it was incubated in 96-well flat-bottom plates, coated by antiBDNF and anti-GDNF monoclonal antibodies. Then, the plates were incubated with polyclonal anti-rabbit antibody for $2 \mathrm{~h}$. For measuring the protein concentration, the color reaction with tetramethyl benzidine was quantified in a plate reader at $450 \mathrm{~nm}$.

\section{Tail Suspension Test (TST)}

The test was performed in a container with posterior, left, and right lateral white walls that a rod $(50 \mathrm{~cm}$ length) was fixed between left and right lateral walls at a distance of $70 \mathrm{~cm}$ from the floor. The mice were hanged in the way that about $1 \mathrm{~cm}$ length of tail end was fixed at the rod using an adhesive tape and left dangling for $6 \mathrm{~min}$. Immobility time was the seconds that hanged mouse remained inactive and immobile. For $6 \mathrm{~min}$, all the movement of mice was recorded by a video camera and analyzed. ${ }^{18}$

\section{Statistical analysis}

Results were expressed as mean \pm S.E.M. The data were analyzed via one way ANOVA with Tukey's post hoc. Nonparametric test of Kruskal-Wallis and Dunn's Multiple Comparisons for post-test. $P<0.05$ was considered as the significance level.

\section{Results}

\section{Effects of FS oil on depressive-like behavior}

TST was used for evolutions of FS oil effects on depressive like behavior. The mean time of immobility was compared between groups. An increased time of immobility was seen Model group $(P<0.05$, Fig. 1). Treatment of normal mice with FS oil (FS group) decreased the mean time of immobility compared to Control group $(P<0.05$, Fig. 1). Also, a decreased mean time of immobility was observed in Model +FS group compared to Model group $(P<0.05$, Fig. 1$)$.

\begin{tabular}{lcl}
\multicolumn{2}{l}{ Table 1. List of primers. } & \\
\hline Gene & \multicolumn{1}{c}{ Forward primer } & \multicolumn{1}{c}{ Reverse Primer } \\
\hline BDNF & CAGGATTGACAGTTTGATAGCTCTATC & ATCGCTCCAGCAACTAACAACG \\
GDNF & AATGTGTCCGTCGTGGATCTG & CAACCTGGTCCTCAGTGTATC \\
b-actin & CTGGGACTACATGGAGAAGAGC & GTCTCAATCACGATCTGGGTCATT \\
\hline
\end{tabular}




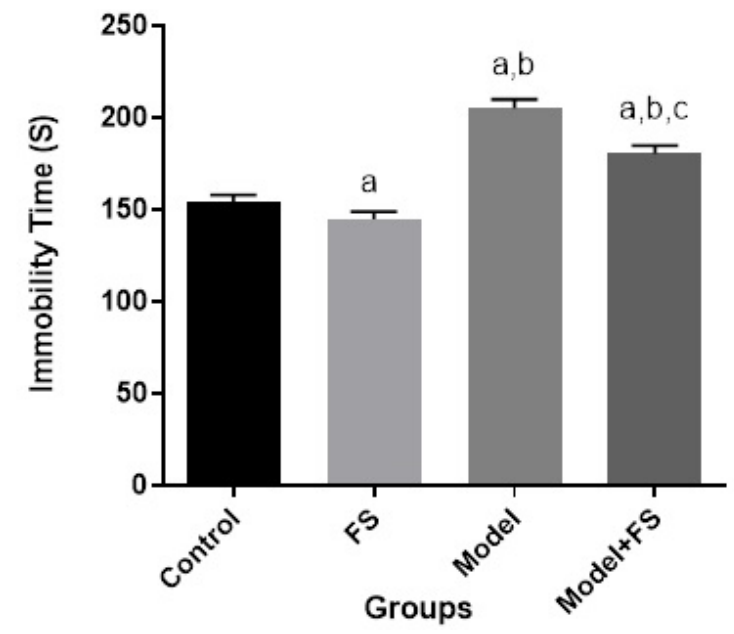

Fig. 1 Effects of treatment with FS oil on immobility time (s) in TST in mice with depressive-like behavior.

$a, P<0.05$ compared to Control group, $b, P<0.05$ compared to FS group, $c, P<0.05$ compared to Model group. Control: Normal mice, FS: Flaxseed oil group (0.2 ml/daily for 20 days), Model: Immobility stress group and received normal saline (20 days), Model+FS: Immobility stress+flaxseed oil.

\section{Effects of FS oil on gene expression of NTFs in CA1 region of mice with depressive-like behavior}

The mean gene expression of NTFs, including DDNF and GDNF was evaluated in CA1 region of hippocampus in each group. According to Fig. 2, there was a significant decrease in the gene expression of BDNF and GDNF in Model group compared to Control and FS groups ( $P<0.05$, Fig. 2a,b). There was a significant increase in the gene expression of BDNF and GDNF in Model+FS group compared with Control, FS, and Model + FS groups $(P<0.05$, Fig. $2 \mathrm{a}, \mathrm{b})$.

\section{Effects of FS oil on protein concentration of NTFs in CA1 region of mice with depressive-like behavior}

The mean protein concentration of NTFs, including DDNF and GDNF was evaluated in CA1 region of hippocampus in each group. Fig. 3 showed that there was a significant decrease in the protein concentration of BDNF and GDNF in Model group compared to Control and FS groups $(P<0.05$, Fig. 3a,b). There was a significant increase in the protein concentration of
BDNF and GDNF in Model+FS group compared to Control, FS, and Model+FS groups $(P<0.05$, Fig. 3a,b).

\section{Effects of FS oil on histopathological alterations of CA1 region of mice with depressive-like behavior}

The mean number of dead neurons was evaluated in the CA1 region of hippocampus in each group. Fig. 4 showed that there was an increase in the number of dead neurons of CA1 region in Model group compared to Control and FS groups $(P<0.05$, Fig. 3a,b). Also, a significant decrease in the number of dead neurons of CA1 region of Model+FS group was recorded compared to Control, FS, and Model+FS groups $(P<0.05$, Fig. 3a,b).

\section{Discussion}

In the present study, we evaluated the effects of FS oil on the depressive-like behavior induced by immobility stress. We used immobility stress to induce depressive like behavior in mice. In this group, the immobility time increased in TST which confirmed the depressive behavior of these animals. Additionally, the number of dead neurons increased and gene expression and protein concentration of NFs decreased in CA1 region of hippocampus. A group of models have been developed to induce depressive-like behaviors in animals through exerting chronic stress. Chronic immobility stress is one of the most convenient models used in preclinical studies in case of time, cost, and energy and its similarity to the available stressors causing depression in human life..$^{19,20}$

Chronic stress is supposed to disrupt oxidant/antioxidant balance and lead to oxidative stress which damage the neuronal cell components such as DNA, plasma membrane and stimulates the inflammatory signaling pathways, results in neuronal death in hippocampal region and depression. ${ }^{21}$ Another studies reported that enhanced inflammation and oxidative stress in brain tissue can lead to depression. ${ }^{22,23} \mathrm{BDNF}$ and GDNF are well-known neurotrophic factors, being in a continuous interaction with serotonergic system. ${ }^{24}$ Reduced levels of NTFs in hippocampus were seen in several neurological disorders. ${ }^{25}$ In the preclinical and clinical studies, the important role of BDNF in depression has been proven. Exposure of animal models to chronic stress may decrease the levels of BDNF in a

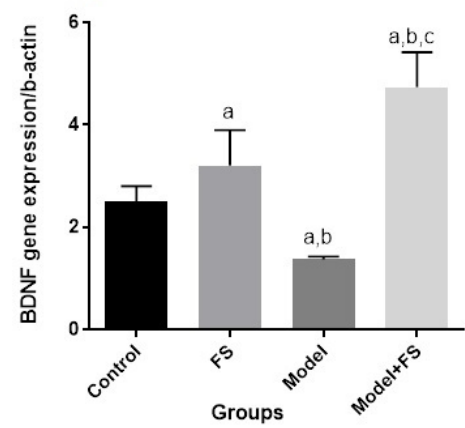

b

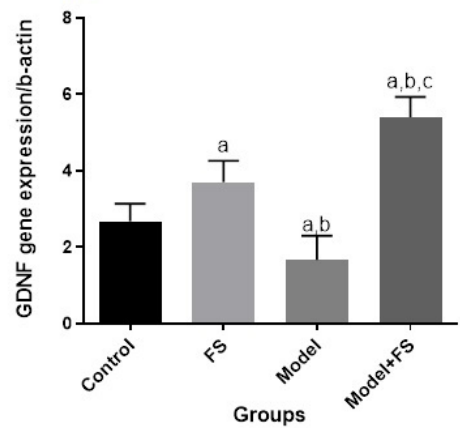

Fig. 2 Effects of FS oil on gene expression of a) BDNF and b) GDNF in CA1 region of mice with depressive-like behavior.

a, $\mathrm{P}<0.05$ compared to Control group, b, P<0.05 compared to FS group, $\mathrm{C}, \mathrm{P}<0.05$ compared to Model group. Control: Normal mice, FS: Flaxseed oil group (0.2 ml/daily for 20 days), Model: Immobility stress group and received normal saline (20 days), Model+FS: Immobility stress+flaxseed oil. 

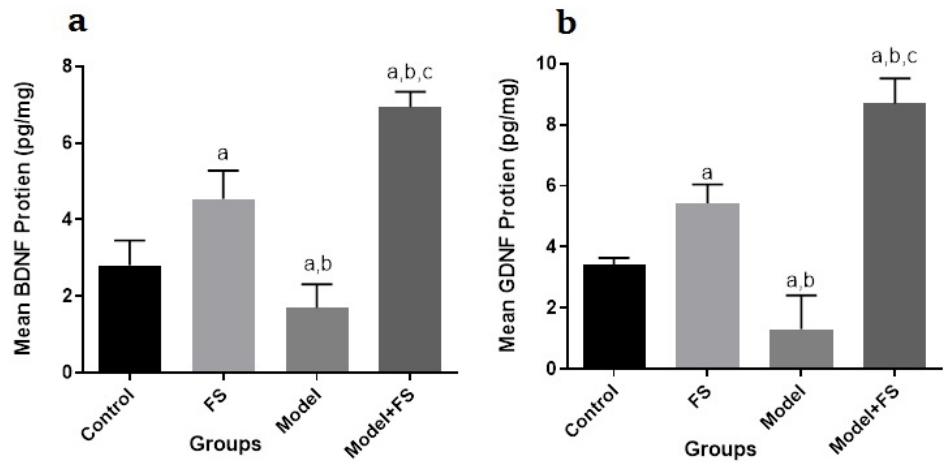

Fig. 3 Effects of FS oil on protein concentration of a) BDNF and b) GDNF in CA1 region of mice with depressive-like behavior.

a, $\mathrm{P}<0.05$ compared to Control group, $\mathrm{b}, \mathrm{P}<0.05$ compared to FS group, $\mathrm{C}, \mathrm{P}<0.05$ compared to Model group. Control: Normal mice, FS: Flaxseed oil group ( $0.2 \mathrm{ml} /$ daily for 20 days), Model: Immobility stress group and received normal saline (20 days), Model+FS: Immobility stress+flaxseed oil.
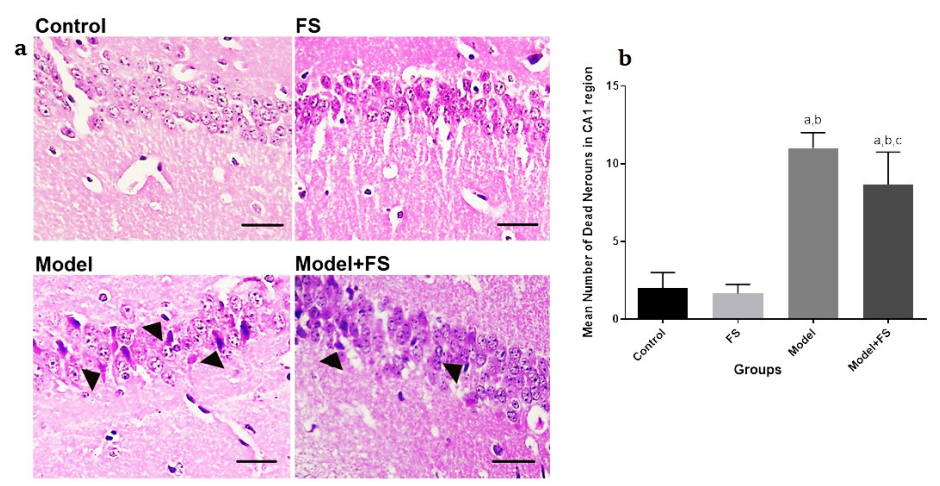

\begin{abstract}
Fig. 4 Effects of $\mathrm{FS}$ oil on histopathological changes of CA1 region of hippocampus of mice with depressive-like behavior.

(a) H\&E staining (scale bar: $50 \mu \mathrm{m}$ ), (b) Comparing the mean number of dead neurons (arrows) of CA1 region in different groups. a, $\mathrm{P}<0.05$ compared to Control group; $b, \mathrm{P}<0.05$ compared to FS group; C, P<0.05 compared to Model group. Control: Normal mice, FS: Flaxseed oil group ( $0.2 \mathrm{ml} /$ daily for 20 days), Model: Immobility stress group and received normal saline (20 days), Model+FS: Immobility stress+flaxseed oil.
\end{abstract}

the hippocampus. ${ }^{8,26}$ On the other hand, treatment of patients with major depressive disorder with antidepressants resulted in alterations in serum BDNF and GNDF levels after 12 weeks. ${ }^{27}$ Targeting the BDNF and GDNF pathways and developing antidepressive strategies to enhance the levels of these NTFs in serum and brain were considered in several studies. ${ }^{28-30}$

Nowadays, it is possible to extract herbal concentrates with acceptable qualities to be used for pharmaceutical purposes. $^{31-33}$ Among a wide range of herbs with beneficial characteristics, FS has been shown to have antioxidant and anti-inflammatory features. ${ }^{34}$ As a functional food, this seed or its oil can be consumed in daily diets. ${ }^{35}$ In the present study, we used oral FS oil for treatment of depressive like disorders following chronic stress. Administration of FS oil for 20 days could decrease the immobility time in TST, reduced dead neurons of CA1 region following induction of depressive behavior. This neuroprotective effects were shown to be related to enhanced levels of NTFs in this critical region. The neuroprotective effects of FS has been demonstrated in several studies. Gholamineghad et al. confirmed the antioxidative and neuroprotective properties of FS in a rat model of spinal ischemic injury. In this study, disrupted functions of rats due to ischemia were significantly improved after treatment with FS daily diet (10\% FS ) for 4 weeks after ischemic surgery. ${ }^{36}$ FS could significantly decreased the levels of inflammatory meditators. ${ }^{37}$ Furthermore, Navaie et al showed the antioxidative, protective, and preventive effects of FS (10\% FS for 4 weeks) on hypoxia-induced hippocampal impairment in rat model..$^{38} \mathrm{FS}$ oil compounds, such as ALA, are capable to enhance the gene expression of neurotrophic factors like BDNF and GDNF. ${ }^{39}$, ${ }^{40}$ Rahmati et al also indicated that FS oil administration $(10 \mathrm{mg} / \mathrm{kg}$ ) for 10 weeks enhanced the levels of BDNS in hippocampus and changed the pain feeling in rat model. ${ }^{41}$ Bagheri et al also reported that FS oil administration $(0.2 \mathrm{ml}$ daily for 3 weeks) also increased the levels of BDNF and GDNF in motor cortex of brain stroke model of rats. ${ }^{42}$

Taken together, FS oil ( $0.2 \mathrm{ml}$ daily for 3 weeks) could decrease the depressive-live disorder via decreasing the neural death in hippocampal CA1 region of mice following a chronic immobility stress for 20 days. Accordingly, it seems that FS oil enhanced the levels of NTFs (BDNF and GDNF) in CA1 region and protected the pyramidal cells of this region against increased levels of inflammatory mediators and oxidative factors, and survived these neurons.

\section{Conflict of Interest}

None 


\section{References}

1. Joo Y, Choi KM, Lee YH, Kim G, Lee DH, Roh GS, et al. Chronic immobilization stress induces anxiety- and depression-like behaviors and decreases transthyretin in the mouse cortex. Neurosci Lett. 2009;461(2):121-5.

2. Sapse AT. Stress, cortisol, interferon and "stress" diseases. I. Cortisol as the cause of "stress" diseases. Med Hypotheses. 1984;13(1):31-44.

3. Carpenter WT, Jr., Gruen PH. Cortisol's effects on human mental functioning. J Clin Psychopharmacol. 1982;2(2):91-101.

4. Martinowich K, Manji H, Lu B. New insights into BDNF function in depression and anxiety. Nat Neurosci. 2007;10(9):1089-93.

5. Riccio A, Ahn S, Davenport CM, Blendy JA, Ginty DD. Mediation by a CREB family transcription factor of NGF-dependent survival of sympathetic neurons. Science. 1999;286(5448):2358-61.

6. Emon MPZ, Das R, Nishuty NL, Shalahuddin Qusar MMA, Bhuiyan MA, Islam MR. Reduced serum BDNF levels are associated with the increased risk for developing MDD: a case-control study with or without antidepressant therapy. BMC Res Notes. 2020;13(1):83.

7. Numakawa T, Adachi N, Richards M, Chiba S, Kunugi H. Brain-derived neurotrophic factor and glucocorticoids: reciprocal influence on the central nervous system. Neuroscience. 2013;239:157-72.

8. Ducray A, Krebs SH, Schaller B, Seiler RW, Meyer M, Widmer HR. GDNF family ligands display distinct action profiles on cultured GABAergic and serotonergic neurons of rat ventral mesencephalon. Brain Res. 2006;1069(1):104-12.

9. Sjors Dahlman A, Blennow K, Zetterberg H, Glise K, Jonsdottir IH. Growth factors and neurotrophins in patients with stress-related exhaustion disorder. Psychoneuroendocrinology. 2019;109:104415.

10. Karege F, Perret G, Bondolfi G, Schwald M, Bertschy G, Aubry JM. Decreased serum brain-derived neurotrophic factor levels in major depressed patients. Psychiatry Res. 2002;109(2):143-8.

11. Liu RJ, Aghajanian GK. Stress blunts serotonin- and hypocretin-evoked EPSCs in prefrontal cortex: role of corticosterone-mediated apical dendritic atrophy. Proc Natl Acad Sci USA. 2008;105(1):359-64

12. Popoli M, Yan Z, McEwen BS, Sanacora G. The stressed synapse: the impact of stress and glucocorticoids on glutamate transmission. Nat Rev Neurosci. 2011;13(1):22-37

13. Yaribeygi H, Panahi Y, Sahraei H, Johnston TP, Sahebkar A. The impact of stress on body function: A review. EXCLI J. 2017;16:1057-72.

14. Popova NK, Ilchibaeva TV, Naumenko VS. Neurotrophic factors (BDNF and GDNF) and the serotonergic system of the brain. Biochemistry (Mosc). 2017;82(3):308-17

15. Bagheri A, Talei S, Hassanzadeh N, Mokhtari T, Akbari M, Malek F, et al. The neuroprotective effects of flaxseed oil supplementation on functional motor recovery in a model of ischemic brain stroke: upregulation of BDNF and GDNF. Acta Med Iran. 2017;55(12):785-92.

16. Connor WE. Alpha-linolenic acid in health and disease. Am J Clin Nutr. 1999;69(5):827-8

17. Tsybko AS, Il'chibaeva TV, Kondaurova EM, Bazovkina DV, Naumenko VS. The effect of central administration of the neurotrophic factors BDNF and GDNF on the functional activity and expression of the serotonin 5-HT2A receptors in mice genetically predisposed to depressive-like behavior. Mol Biol (Mosk). 2014;48(6):983-9.

18. Can A, Dao DT, Terrillion CE, Piantadosi SC, Bhat S, Gould TD. The tail suspension test. J Vis Exp. 2012(59):e3769.

19. Son $\mathrm{H}$, Yang JH, Kim HJ, Lee DK. A chronic immobilization stress protocol for inducing depression-like behavior in mice. JoVE (J of Visual Exp). 2019(147):e59546.

20. Liu L, Zhou X, Zhang Y, Liu Y, Yang L, Pu J, et al. The identification of metabolic disturbances in the prefrontal cortex of the chronic restraint stress rat model of depression. Behav Brain Res. 2016:305:148-56.

21. Duman RS. Neuronal damage and protection in the pathophysiology and treatment of psychiatric illness: stress and depression. Dialogues Clin Neurosci. 2009;11(3):239-55

22. Yanik M, Erel O, Kati M. The relationship between potency of oxidative stress and severity of depression. Acta Neuropsychiatrica. 2004;16(4):200-3.

23. Lopresti AL, Maker GL, Hood SD, Drummond PD. A review of peripheral biomarkers in major depression: the potential of inflammatory and oxidative stress biomarkers. Prog Neuro-Psychopharmacol Biol Psychiatry. 2014:48:102-11.

24. Popova N, Ilchibaeva T, Naumenko V. Neurotrophic factors (BDNF and GDNF) and the serotonergic system of the brain. Biochemistry (Moscow). 2017;82(3):308-17
25. Mokhtari T, Akbari M, Malek F, Kashani IR, Rastegar T, Noorbakhsh F, et al. Improvement of memory and learning by intracerebroventricular microinjection of T3 in rat model of ischemic brain stroke mediated by upregulation of BDNF and GDNF in CA1 hippocampal region. DARU J Pharm Sci. 2017;25(1):4.

26. Tsankova NM, Berton O, Renthal W, Kumar A, Neve RL, Nestler EJ. Sustained hippocampal chromatin regulation in a mouse model of depression and antidepressant action. Nat Neurosci. 2006;9(4):519-25.

27. Park Y-M, Lee B-H. Alterations in serum BDNF and GDNF levels after 12 weeks of antidepressant treatment in female outpatients with major depressive disorder. Psychiatry Investig. 2018;15(8):818-23.

28. Angelucci F, Aloe L, Jiménez-Vasquez P, Mathé AA. Lithium treatment alters brain concentrations of nerve growth factor, brain-derived neurotrophic factor and glial cell line-derived neurotrophic factor in a rat model of depression. Int J Neuropsychopharmacol. 2003;6(3):225-31.

29. Tunca Z, Ozerdem A, Ceylan D, Yalçın Y, Can G, Resmi H, et al. Alterations in BDNF (brain derived neurotrophic factor) and GDNF (glial cell line-derived neurotrophic factor) serum levels in bipolar disorder: the role of lithium. J Affect Disorders. 2014:166:193-200.

30. Varela RB, Valvassori SS, Lopes-Borges J, Mariot E, Dal-Pont GC, Amboni RT, et al. Sodium butyrate and mood stabilizers block ouabain-induced hyperlocomotion and increase BDNF, NGF and GDNF levels in brain of Wistar rats. J Psychiatric Res. 2015;61:114-21.

31. Ashaari Z, Hadjzadeh M-A-R, Hassanzadeh G, Alizamir T, Yousefi B, Keshavarzi Z, et al. The flavone luteolin improves central nervous system disorders by different mechanisms: a review. J Mol Neurosci. 2018;65(4): 491-506.

32. Ghaffari N, Hassanzadeh G, Nowrouzi A, Gholaminejhad M, Mokhtari T, Seifali R, et al. Antioxidative and anti-inflammatory effects of Cichorium intybus $L$. seed extract in ischemia/reperfusion injury model of rat spinal cord. J Contemp Med Sci. 2018;4(4).

33. Mokhtari T, Hussein Osman H-E, El-Meghawry El-Kenawy A, Dashti N. Ameliorative effect of virgin olive oil against nephrotoxicity following subchronic administration of ethephon in male rats. J Tradit Complement Med. 2020;10(5):487-95.

34. Mokhtari T, Faghir Ghanesefat H, Hassanzadeh G, Moayeri A, Haeri SMJ, Rezaee Kanavee A, et al. Effects of Flaxseed oil supplementation on renal dysfunction due to ischemia/reperfusion in rat. Ilam Univ Med Sci. 2017:4(1):22-9.

35. Goyal A, Sharma V, Upadhyay N, Gill S, Sihag M. Flax and flaxseed oil: an ancient medicine \& modern functional food. J Food Sci Technol. 2014:51(9):1633-53.

36. Gholaminejhad M, Arabzadeh S, Akbari M, Mohamadi Y, Hassanzadeh G Anti-oxidative and neuroprotective effects of flaxseed on experimental unilateral spinal cord injury in rat. J Contemp Med Sci. 2017;3(10):213-7.

37. Gholaminejhad M, Hassanzadeh G, Akbari M. O2: flaxseed reduces proinflammatory factors IL-1 $\beta, I L-18$ and TNF-a in injured spinal cord rat model. Shafaye Khatam Neurosci J 2018;6(2):2.

38. Navaie F, Hassanzadeh G, Mahakizadeh S, Mehrannia K, Alizamir T, Dashti N, et al. Anti-oxidative and neuroprotective effects of supplementary flaxseed on oxidative damage in the hippocampus area of a rat model of hypoxia. Arch Neurosci. 2018;5(4)

39. Hadjighassem M, Kamalidehghan B, Shekarriz N, Baseerat A, Molavi N, Mehrpour $\mathrm{M}$, et al. Oral consumption of a-linolenic acid increases serum BDNF levels in healthy adult humans. Nutr J. 2015;14(1):20.

40. Razi SS, Latif MJ, Li X, Afthinos JN, Ippagunta N, Schwartz G, et al. Dietary flaxseed protects against lung ischemia reperfusion injury via inhibition of apoptosis and inflammation in a murine model. J Surg Res. 2011;171(1):e113-e21.

41. Rahmati-Ahmadabad S, Azarbayjani M, Nasehi M. The effects of highintensity interval training with supplementation of flaxseed oil on BDNF mRNA expression and pain feeling in male rats. Ann Appl Sport Sci. 2017:5(4):1-12.

42. Bagheri A, Talei S, Hassanzadeh N, Mokhtari T, Akbari M, Malek F, et al. The neuroprotective effects of flaxseed oil supplementation on functional motor recovery in a model of ischemic brain stroke: upregulation of BDNF and GDNF. Acta Med Iran. 2017:785-92.

This work is licensed under a Creative Commons Attribution-NonCommercial 3.0 Unported License which allows users to read, copy, distribute and make derivative works for non-commercial purposes from the material, as long as the author of the original work is cited properly. 